\title{
On extending the Quantum Measure
}

\author{
Fay Dowker ${ }^{1}$, Steven Johnston ${ }^{1}$, Sumati Surya ${ }^{2}$ \\ ${ }^{1}$ Blackett Laboratory, Imperial College, London, SW7 2AZ, UK \\ ${ }^{2}$ Raman Research Institute, Sadashivanagar, Bangalore, 560080, India
}

October 30, 2018

\begin{abstract}
We point out that a quantum system with a strongly positive quantum measure or decoherence functional gives rise to a vector valued measure whose domain is the algebra of events or physical questions. This gives an immediate handle on the question of the extension of the decoherence functional to the sigma algebra generated by this algebra of events. It is on the latter that the physical transition amplitudes directly give the decoherence functional. Since the full sigma algebra contains physically interesting questions, like the return question, extending the decoherence functional to these more general questions is important. We show that the decoherence functional, and hence the quantum measure, extends if and only if the associated vector measure does. We give two examples of quantum systems whose decoherence functionals do not extend: one is a unitary system with finitely many states, and the other is a quantum sequential growth model for causal sets. These examples fail to extend in the formal mathematical sense and we speculate on whether the conditions for extension are unphysically strong.
\end{abstract}

\section{Introduction}

The need for a measurement independent interpretation of quantum theory is perhaps most keenly felt when constructing a theory of quantum cosmology. In describing the physics of the very early universe we are confronted with the dilemma of how to interpret the quantum formalism in the absence of external measurements. The standard interpretation places an emphasis on the state vector, and gives primary status to external measurements but it is questions of spacetime form that are of interest in quantum cosmology e.g. how likely is it for a homogeneous and isotropic universe to arise from an initial big-bang type singularity?

Quantum Measure Theory [1, 2, 3, 4, 5, 6, is a formulation of quantum theory based on the path integral. Since measuring devices play no fundamental role in 
this approach, it is ideally suited to examining theories of quantum cosmology. Quantum Measure Theory gives conceptual primacy to the sample space of histories or spacetime configurations, $\Omega$, which is summed over in the path integral and takes its cue from the measure theoretic formulation of classical stochastic dynamics. A physical quantum system is described by a quantum measure space, $(\Omega, \mathfrak{A}, \mu)$, where $\mathfrak{A}$ is an event algebra or set of propositions about the system and dynamical information is contained in the quantum measure $\mu: \mathfrak{A} \rightarrow \mathbb{R}^{+}$which is given by the path integral. $\mu$ obeys the quantum sum rule [1]

$$
\mu(\alpha \cup \beta \cup \gamma)=\mu(\alpha \cup \beta)+\mu(\alpha \cup \gamma)+\mu(\beta \cup \gamma)-\mu(\alpha)-\mu(\beta)-\mu(\gamma)
$$

all for pairwise disjoint sets $\alpha, \gamma, \beta \in \mathfrak{A} . \mu$ is not in general a probability measure (even with the normalisation $\mu(\Omega)=1$ ) since it does not satisfy the Kolmogorov sum rule in the presence of quantum interference: $\exists \alpha, \beta \in \mathfrak{A}$ with $\alpha \cap \beta=\emptyset$ and $\mu(\alpha \cup \beta) \neq \mu(\alpha)+\mu(\beta)$. Thus, Quantum Measure Theory is a genuine generalisation of classical stochastic dynamics.

In classical stochastic theories, the probability measure on the event algebra is often defined indirectly in terms of transition probabilities from one momentary state to another. The classical random walk is a standard example. The transition probabilities define, directly, the probability of events which are limited in time, for example: "Is the walker at site $x$ at time $t$ ?" Given an initial position at say $t=0$, one calculates the probabilities of each of the walks with $t$ steps that end at $x$ using the transition probabilities and adds them together. The probability of certain physically interesting events, however, cannot be directly calculated in this way. These questions involve arbitrarily long times and are epitomised by the return question: "Does the walker ever return to the origin?" In order to find the probability of such events, one must be able to extend the probability measure on the finite-time events to infinite-time events. For non-negative measures this is guaranteed by the Carathéodory-Kolmogorov extension theorem, which gives a unique extension of the measure on the algebra of finite-time events to the sigma algebra it generates. The sigma algebra is closed under countable unions and intersections and one can show that the "return event" is an element of this algebra 7, 8,

Similarly, in most quantum systems the quantum measure derives from transition amplitudes from one momentary state to another. To make predictions about infinite-time events, like the quantum analogue of the return question, requires an extension of the quantum measure to an algebra that includes such events. There is however no known analog of the Carathéodory-Kolmogorov extension theorem for quantum measures. In this paper we take first steps in investigating this issue. The technical development that helps this analysis is the histories Hilbert space construction from quantum measures which derive from a decoherence functional [9. We show that the quantum measure is equivalent to a derived vector measure, i.e. a measure valued in this histories Hilbert space. Unlike the quantum measure, vector measures are additive and have been studied extensively in the literature [10. In this paper, we address the question 
of extension of the quantum measure by studying the derived vector measure for a class of systems in which the histories Hilbert space is finite dimensional.

In Section 2 we define the quantum vector "pre-measure" on the algebra of finite-time events after reviewing the histories Hilbert space construction of 9. In Section 3 we examine finite dimensional unitary systems which evolve in discrete time steps, and show that for a generic evolution, the quantum vector pre-measure does not extend to a quantum vector measure. In Section 4 we define the complex percolation sequential growth dynamics for causal sets and show that the quantum vector pre-measure does not extend except when the amplitudes are real and non-negative. In Section 5 we discuss the implications of our results. For the complex percolation type models, the question of an extension is tied closely to the construction of covariant observables. Using an example we show that the lack of an extension is related to other pathologies.

\section{The Quantum Vector Measure}

In this section we show that a quantum measure space in which the quantum measure derives from a strongly positive decoherence functional is equivalent to a vector measure which takes its values in a Hilbert space.

In order to formulate quantum dynamics as a measure space, the sample space $\Omega$ is taken to be the set of histories summed over in the path integral. For a single particle in $\mathbb{R}^{3}$ whose evolution starts at some initial time this is the space of all trajectories that are infinite to the future, while for a scalar field in $\mathbb{R}^{3} \times \mathbb{R}$ it is the set of all spacetime field configurations. An event algebra $\mathfrak{A}$ over the sample space $\Omega$ is a collection of subsets of $\Omega$ that forms an algebra or field of sets over $\Omega$. Thus, (i) $\alpha \in \mathfrak{A} \Rightarrow \alpha^{c} \in \mathfrak{A}$, where $\alpha^{c}$ is the complement of $\alpha$ in $\Omega$ and (ii) $\alpha \cap \beta \in \mathfrak{A}$ and $\alpha \cup \beta \in \mathfrak{A}$ for any $\alpha, \beta \in \mathfrak{A}$. A sigma algebra $\mathfrak{S}$ satisfies, in addition to (i) and (ii), closure under countable unions. The sigma algebra $\mathfrak{S}_{\mathfrak{A}}$ generated by an algebra $\mathfrak{A}$ is defined to be the (unique) smallest sigma algebra containing $\mathfrak{A}$. In what follows, in order to distinguish between a measure on an algebra and that on a sigma algebra, we will refer to the former as a pre-measure and the latter as a measure (thus, a pre-measure is a measure if the algebra on which it is defined is a sigma algebra).

A decoherence functional is a complex function $D: \mathfrak{A} \times \mathfrak{A} \rightarrow \mathbb{C}$ which represents the quantum interference between two events. $D$ is 4$]$

1. Hermitian: For all $\alpha, \beta \in \mathfrak{A}$, we have $D(\alpha, \beta)=D^{*}(\beta, \alpha)$.

2. Finitely bi-additive: For any $\alpha \in \mathfrak{A}$ and $m$ mutually disjoint $\beta_{i} \in \mathfrak{A}$, we have $D\left(\alpha, \cup_{i=1}^{m} \beta_{i}\right)=\sum_{i=1}^{m} D\left(\alpha, \beta_{i}\right)$. Similarly, for any $\beta \in \mathfrak{A}$ and $m$ mutually disjoint $\alpha_{i} \in \mathfrak{A}, D\left(\cup_{i=1}^{m} \alpha_{i}, \beta\right)=\sum_{i=1}^{m} D\left(\alpha_{i}, \beta\right)$.

3. Normalised: $D(\Omega, \Omega)=1$.

4. Strongly positive: For any finite collection of $\left\{\alpha_{i}\right\}$ in $\mathfrak{A}$, the matrix $M_{i j} \equiv$ $D\left(\alpha_{i}, \alpha_{j}\right)$ is positive semi-definite, i.e., it has non-negative eigenvalues. 
The quantum pre-measure $\mu: \mathfrak{A} \rightarrow \mathbb{R}^{+}$derives from the decoherence functional via $\mu(\alpha) \equiv D(\alpha, \alpha)$ and we see that the biadditivity of $D$ means that $\mu$ satisfies the quantum sum rule (11) but $\mu(\alpha \cup \beta) \neq \mu(\alpha)+\mu(\beta)$ if $\operatorname{Re}(D(\alpha, \beta)) \neq 0$, for disjoint $\alpha$ and $\beta$. In what follows we will refer interchangeably to both $D$ and $\mu$ as the quantum pre-measure]. The construction in [9] of a Hilbert space from the event algebra $\mathfrak{A}$ and the decoherence functional $D$ implies that the quantum measure is equivalent to a Hilbert space valued measure which is additive, unlike the quantum measure 2. This gives us a useful "vector measure" avatar of the quantum measure. We now briefly review vector measures and pre-measures.

A vector pre-measure [10] $\eta_{\mathrm{v}}$ is a function from an algebra $\mathfrak{A}$ over $\Omega$ to a Banach space $\mathcal{B}$ which is finitely additive, i.e., for every disjoint pair $\alpha, \beta \in \mathfrak{A}$

$$
\eta_{\mathrm{v}}(\alpha \cup \beta)=\eta_{\mathrm{v}}(\alpha)+\eta_{\mathrm{v}}(\beta) .
$$

If $\mathfrak{S}$ is a sigma algebra, a vector measure $\overline{\eta_{\mathrm{v}}}: \mathfrak{S} \rightarrow \mathcal{B}$ is moreover required to be countably additive

$$
\overline{\eta_{v}}\left(\bigcup_{n=1}^{\infty} \alpha_{n}\right)=\sum_{n=1}^{\infty} \overline{\eta_{\mathrm{v}}}\left(\alpha_{n}\right)
$$

in the norm topology of $\mathcal{B}$, for all sequences $\alpha_{n}$ of pairwise disjoint members of $\mathfrak{S}$. The sum $\sum_{n=1}^{\infty} \overline{\eta_{\mathrm{v}}}\left(\alpha_{n}\right)$ must therefore converge unconditionally in the norm.

The Banach space of interest to us is the histories Hilbert space $\mathcal{H}$ of 9 and we briefly review this construction below. Let $V$ be the space of complex valued functions on $\mathfrak{A}$ which are non-zero only on a finite number of elements of $\mathfrak{A}$. $V$ is the free vector space over $\mathfrak{A}$ and the decoherence functional provides an inner product

$$
\langle u, v\rangle_{V} \equiv \sum_{\alpha \in \mathfrak{A}} \sum_{\beta \in \mathfrak{A}} u^{*}(\alpha) v(\beta) D(\alpha, \beta) .
$$

$V$ is itself not a Hilbert space since it contains zero-norm vectors and may not be complete. The histories Hilbert space $\mathcal{H}$ is constructed by taking the set of Cauchy sequences $\left\{u_{i}\right\}$ in $V$ and quotienting by the equivalence relation

$$
\left\{u_{i}\right\} \sim\left\{v_{i}\right\} \quad \text { if } \quad \lim _{i \rightarrow \infty}\left\|u_{i}-v_{i}\right\|_{V}=0
$$

where the norm is given by the inner product. We have

$$
\begin{aligned}
{\left[\left\{u_{i}\right\}\right]+\left[\left\{v_{i}\right\}\right] } & \equiv\left[\left\{u_{i}+v_{i}\right\}\right] \\
\lambda\left[\left\{u_{i}\right\}\right] & \equiv\left[\left\{\lambda u_{i}\right\}\right], \\
\left\langle\left[\left\{u_{i}\right\}\right],\left[\left\{v_{i}\right\}\right]\right\rangle & \equiv \lim _{i \rightarrow \infty}\left\langle u_{i}, v_{i}\right\rangle_{V}
\end{aligned}
$$

for all $\left[\left\{u_{i}\right\}\right],\left[\left\{v_{i}\right\}\right] \in \mathcal{H}, \lambda \in \mathbb{C}$.

\footnotetext{
${ }^{1}$ While $\mu$ has no standard measure theoretic analogue, since it is not additive, the decoherence functional belongs to the class of "biadditive complex-valued pre-measures", also called "bi-measures" or "poly-measures" [11].

${ }^{2}$ We thank Rafael Sorkin for this observation.
} 
We define the quantum vector pre-measure $\mu_{\mathrm{v}}: \mathfrak{A} \rightarrow \mathcal{H}$ to be

$$
\mu_{\mathrm{v}}(\alpha) \equiv\left[\chi_{\alpha}\right] \in \mathcal{H} .
$$

where [.] denotes the equivalence class under (5D) and we use the shorthand $\chi_{\alpha}$ to denote the constant Cauchy sequence $\left\{\chi_{\alpha}\right\}$ for the indicator function $\chi_{\alpha}: \mathfrak{A} \rightarrow\{0,1\}$

$$
\chi_{\alpha}(\beta)= \begin{cases}1 & \text { if } \beta=\alpha, \\ 0 & \text { if } \beta \neq \alpha .\end{cases}
$$

Thus,

$$
\left\langle\mu_{\mathrm{v}}(\alpha), \mu_{\mathrm{v}}(\beta)\right\rangle=D(\alpha, \beta),
$$

with the inner product taken in $\mathcal{H}$. The bi-additivity of $D$ means that for any disjoint pair, $\alpha, \beta \in \mathfrak{A}$ we have

$$
\left\|\chi_{\alpha \cup \beta}-\chi_{\alpha}-\chi_{\beta}\right\|=0
$$

in the norm derived from the inner product (44). This ensures that $\mu_{\mathrm{v}}(\alpha \cup \beta)=$ $\mu_{\mathrm{v}}(\alpha)+\mu_{\mathrm{v}}(\beta)$ and therefore that $\mu_{\mathrm{v}}$ is finitely additive:

$$
\mu_{\mathrm{v}}\left(\bigcup_{i=1}^{n} \alpha_{i}\right)=\sum_{i=1}^{n} \mu_{\mathrm{v}}\left(\alpha_{i}\right),
$$

for $n$ mutually disjoint sets $\alpha_{i} \in \mathfrak{A}$. Hence $\mu_{\mathrm{v}}$ is a vector pre-measure.

We pause here to note that the term "quantum vector (pre-)measure" does not imply that $\mu_{v}$ fails to satisfy the Kolmogorov sum rule $-\mu_{v}$ is a vector pre-measure and is finitely additive. We use the phrase "quantum vector (pre)measure" to emphasise the physical origins of $\mu_{\mathrm{v}}$ and that it is valued in the histories Hilbert space $\mathcal{H}$ constructed from the quantum measure.

As discussed in the introduction, an important question in measure theory is whether a pre-measure defined on an algebra $\mathfrak{A}$ extends to a measure on the sigma algebra $\mathfrak{S}_{\mathfrak{A}}$ generated by $\mathfrak{A}$. Specifically a vector measure $\bar{\eta}_{\mathrm{v}}: \mathfrak{S}_{\mathfrak{A}} \rightarrow \mathcal{B}$ is said to be an extension of a vector pre-measure $\eta_{\mathrm{v}}: \mathfrak{A} \rightarrow \mathcal{B}$, if its restriction to $\mathfrak{A} \subset \mathfrak{S}$ is $\left.\bar{\eta}_{\mathrm{v}}\right|_{\mathfrak{A}}=\eta_{\mathrm{v}}$. A vector pre-measure $\eta_{\mathrm{v}}$ on $\mathfrak{A}$ is then said to extend to $\mathfrak{S}_{\mathfrak{A}}$ if there exists a unique vector measure $\overline{\eta_{\mathrm{v}}}: \mathfrak{S}_{\mathfrak{A}} \rightarrow \mathcal{B}$ such that $\left.\bar{\eta}_{\mathrm{v}}\right|_{\mathfrak{A}}=\eta_{\mathrm{v}}$.

The extension $\overline{\mu_{\mathrm{v}}}$ of a quantum vector pre-measure $\mu_{\mathrm{v}}$, if it exists, can be used to define a decoherence functional $\bar{D}: \mathfrak{S}_{\mathfrak{A}} \times \mathfrak{S}_{\mathfrak{A}} \rightarrow \mathbb{C}$

$$
\bar{D}(\alpha, \beta) \equiv\left\langle\overline{\mu_{\mathrm{v}}}(\alpha), \overline{\mu_{\mathrm{v}}}(\beta)\right\rangle \quad \forall \alpha, \beta \in \mathfrak{S}_{\mathfrak{A}} .
$$

The restriction $\left.\bar{D}\right|_{\mathfrak{A}}=D$, the decoherence functional on $\mathfrak{A}$, and hence $\bar{D}$ can be viewed as an extension of $D$ on $\mathfrak{A} \times \mathfrak{A}$ to a functional on $\mathfrak{S}_{\mathfrak{A}} \times \mathfrak{S}_{\mathfrak{A}} . \bar{D}$ is Hermitian, since

$$
\left\langle\overline{\mu_{\mathrm{v}}}(\alpha), \overline{\mu_{\mathrm{v}}}(\beta)\right\rangle=\left\langle\overline{\mu_{\mathrm{v}}}(\beta), \overline{\mu_{\mathrm{v}}}(\alpha)\right\rangle^{*} .
$$

Moreover, the countable additivity of $\overline{\mu_{\mathrm{v}}}$ and the countable biadditivity of the inner product implies countable biadditivity of $\bar{D}$. In addition, $\bar{D}$ is strongly positive. Finally, since $\Omega \in \mathfrak{A}, \bar{D}(\Omega, \Omega)=D(\Omega, \Omega)=1$. Thus, the extension 
$\mu_{\mathrm{v}}$ can be used to construct a countably biadditive, positive and Hermitian decoherence functional on $\mathfrak{S}_{\mathfrak{A}}$. If $\overline{\mu_{\mathrm{v}}}$ is the unique extension of $\mu_{\mathrm{v}}$, then so is $\bar{D}$ the unique extension of $D$. (On the other hand, a unique extension $\bar{D}$ of $D$ yields a $\overline{\mu_{v}}$ determined only up to an overall phase which itself can be determined if $\mu_{\mathrm{v}}$ is also known.)

For a non-negative scalar pre-measure $\mu: \mathfrak{A} \rightarrow \mathbb{R}^{+}$, the Carathéodory extension theorem [7, 8] guarantees the existence of a unique extension. For complex vector measures the Carathéodory-Hahn-Kluvanek extension theorem [10] gives necessary and sufficient conditions for a vector pre-measure on $\mathfrak{A}$ to extend to a vector measure on $\mathfrak{S}_{\mathfrak{A}}$. In the case of finite dimensional vector measures, the extension question is equivalent to the simpler question of the extension of the complex scalar component measures. Since our present interest is in examining finite dimensional systems, we will not discuss the CarathéodoryHahn-Kluvanek theorem here, though it may be relevant to the larger program. For our purposes it suffices to focus on the property of bounded variation. The total variation $\left|\mu_{\mathrm{v}}\right|$ of a vector pre-measure $\mu_{\mathrm{v}}$ is defined to be

$$
\left|\mu_{\mathrm{v}}\right|(\alpha)=\sup _{\pi(\alpha)} \sum_{\rho}\left\|\mu_{\mathrm{v}}\left(\alpha_{\rho}\right)\right\|,
$$

where the supremum is over all finite partitions $\pi(\alpha)=\left\{\alpha_{\rho}\right\}$ of $\alpha$ (note that $\left|\mu_{\mathrm{v}}\right|(\alpha)$ is not just $\left.\left|\mu_{\mathrm{v}}(\alpha)\right|\right)$. $\left|\mu_{\mathrm{v}}\right|$ is itself a non-negative finitely additive premeasure on $\mathfrak{A}$ and is countably additive iff $\mu_{\mathrm{v}}$ is (Prop. 9, Chapter 1.1, [10]). $\mu_{\mathrm{v}}$ is said to be of bounded variation if $\left|\mu_{\mathrm{v}}\right|(\alpha)<\infty$ for all $\alpha \in \mathfrak{A}$.

We note that in any basis the components $\mu_{\mathrm{v}}^{(i)}, i=1, \ldots, n$ of a vector pre-measure $\mu_{\mathrm{v}}: \mathfrak{S} \rightarrow \mathbb{C}^{n}$ are themselves complex-valued pre-measures on $\mathfrak{S}$.

Claim 1 Let $\mu_{\mathrm{v}}: \mathfrak{A} \rightarrow \mathbb{C}^{n}$ be a vector pre-measure and $\mu_{\mathrm{v}}{ }^{(i)}: \mathfrak{A} \rightarrow \mathbb{C}, i=$ $1, \ldots n$ be the components of $\mu_{\mathrm{v}}$ in an orthonormal basis. Then $\mu_{\mathrm{v}}$ is of bounded variation iff $\mu_{\mathrm{v}}{ }^{(i)}$ is of bounded variation.

Proof: Since $\left\|\mu_{\mathrm{v}}(\alpha)\right\| \geq\left\|\mu_{\mathrm{v}}{ }^{(i)}(\alpha)\right\|$ for every $i \in\{1, \ldots, n\}$,

$$
\left|\mu_{\mathrm{v}}\right|(\alpha) \geq\left|\mu_{\mathrm{v}}{ }^{(i)}\right|(\alpha)
$$

for each $i$. Therefore if $\mu_{\mathrm{v}}$ is of bounded variation then so is $\mu_{\mathrm{v}}{ }^{(i)}$. From the triangle inequality

$$
\left\|\mu_{\mathrm{v}}(\alpha)\right\| \leq \sum_{i=1}^{n}\left|\mu_{\mathrm{v}}{ }^{(i)}(\alpha)\right|
$$

Thus, for any finite partition $\left\{\alpha_{\rho}\right\}$ of $\alpha, \rho \in\{1, \ldots, m<\infty\}$

$$
\sum_{\rho=1}^{m}\left\|\mu_{\mathrm{v}}\left(\alpha_{\rho}\right)\right\| \leq \sum_{\rho=1}^{m} \sum_{i=1}^{n}\left|\mu_{\mathrm{v}}{ }^{(i)}\left(\alpha_{\rho}\right)\right| \leq \sum_{i=1}^{n}\left|\mu_{\mathrm{v}}{ }^{(i)}(\alpha)\right|,
$$

Clearly, if $\left|\mu_{\mathrm{v}}{ }^{(i)}\right|(\alpha) \leq b_{i}<\infty$, i.e., it is bounded for each $i$, then

$$
\sum_{\rho=1}^{m}\left\|\mu_{\mathrm{v}}\left(\alpha_{\rho}\right)\right\|<\sum_{i} b_{i}
$$


for every finite partition of $\alpha$. Hence $\mu_{\vee}$ is also bounded.

For a complex measure countable additivity implies that its total variation is bounded [12. The components ${\overline{\mu_{\mathrm{v}}}}^{(i)} i=1, \ldots, n$ of a countably additive vector measure $\overline{\mu_{\mathrm{v}}}: \mathfrak{S} \rightarrow \mathbb{C}^{n}$ are also countably additive, and hence are of bounded variation. Thus, by the above Claim, the countable additivity of $\overline{\mu_{\mathrm{v}}}$ implies that it is of bounded variation.

Bounded variation of a complex measure implies its restriction $\left.\mu\right|_{\mathfrak{A}}$ to any subalgebra $\mathfrak{A} \subset \mathfrak{S}$ is also of bounded variation. Thus, a necessary condition for a complex pre-measure on $\mathfrak{A}$ to extend to a measure on $\mathfrak{S}_{\mathfrak{A}}$ is that it is of bounded variation. Along with the above results this means that

Claim 2 Bounded variation is a necessary condition for a finite dimensional vector pre-measure $\mu_{\mathrm{v}}: \mathfrak{A} \rightarrow \mathbb{C}^{N}$ to extend to a vector measure $\overline{\mu_{\mathrm{v}}}: \mathfrak{S}_{\mathfrak{A}} \rightarrow \mathbb{C}^{N}$.

\section{$3 \quad$ Finite Unitary Systems}

In this section we consider the class of finite $N$ dimensional systems which evolve unitarily in discrete unit time steps, $t=1,2,3 \ldots$ In the standard Hilbert space formulation, the Hilbert space at time $t=m$ is $\mathcal{H}_{m}=\mathbb{C}^{N}$. The evolution of a state $\psi \in \mathcal{H}_{1}$ at an initial time $t=1$ to a state $\psi_{m} \in \mathcal{H}_{m}$ at time $t=m$ is governed by the $N \times N$ single-step unitary matrices $U(k+1, k)$ :

$$
\psi_{m}=U(m, m-1) U(m-1, m-2) \ldots U(2,1) \psi .
$$

Let $\left\{e_{1}, e_{2}, \ldots e_{N}\right\}$ be an orthonormal basis for $\mathcal{H}_{1}$.

To describe this system as a quantum measure space we first identify a history as an infinite string $\gamma^{(\infty)}=\left(s_{1}, s_{2}, \ldots, s_{i}, \ldots\right)$, where each entry $s_{i} \in$ $\{1, \ldots, N\}$. The configuration space has $N$ sites, each associated with one of the basis vectors $e_{s} . \Omega$ is the infinite collection of all such strings and the event algebra $\mathfrak{A}$ is generated as follows. We associate with every length $m$ finite string $\gamma=\left(s_{1}, s_{2} \ldots s_{m}\right)$ a cylinder set

$$
\operatorname{cyl}(\gamma) \equiv\left\{\gamma^{(\infty)} \in \Omega \mid \gamma^{(\infty)}(i)=s_{i}, i=1,2, \ldots m\right\},
$$

which is the set of histories for which the first $m$ entries are specified by the string $\gamma$ but are unspecified thereafter. If $\gamma=\left(s_{1}, s_{2}, \ldots, s_{m}\right)$ and $\gamma^{\prime}=\left(s_{1}^{\prime}, s_{2}^{\prime}, \ldots, s_{m}^{\prime}\right)$, with $m^{\prime}>m$, then

$$
\begin{array}{cl}
\operatorname{cyl}\left(\gamma^{\prime}\right) \subset \operatorname{cyl}(\gamma) & \text { if } s_{i}=s_{i}^{\prime} \forall i \in\{1,2, \ldots m\} \\
\operatorname{cyl}\left(\gamma^{\prime}\right) \cap \operatorname{cyl}(\gamma)=\emptyset & \text { otherwise. }
\end{array}
$$

If $\mathfrak{A}$ represents the algebra generated from finite unions and intersections of these cylinder sets, then (20) and (21) imply that any $\alpha \in \mathfrak{A}$ can be expressed as a finite disjoint union of cylinder sets. In particular, for every $\alpha \in \mathfrak{A}$ there exists an $m$ and a $k \leq N^{m}$ such that $\alpha=\cup_{i=1}^{k} \operatorname{cyl}\left(\gamma_{k}\right)$ where the $\gamma_{k}$ are strings 
of length $m$ and the $\operatorname{cyl}\left(\gamma_{k}\right)$ are mutually disjoint. Here, $N^{m}$ is the number of possible length $m$ strings.

The decoherence functional for such a unitary system is given by

$$
D\left(\operatorname{cyl}(\gamma), \operatorname{cyl}\left(\gamma^{\prime}\right)\right)=A^{*}(\gamma) A\left(\gamma^{\prime}\right) \delta_{s_{m} s_{m}^{\prime}},
$$

where $\gamma, \gamma^{\prime}$ are length $m$ finite strings (or "truncated histories") and $A\left(\gamma_{i}\right)$ is a complex amplitude. Assuming an initial state $\psi \in \mathcal{H}_{1}$

$$
A(\operatorname{cyl}(\gamma))=U_{s_{m} s_{m-1}}(m, m-1) \ldots U_{s_{3} s_{2}}(3,2) U_{s_{2} s_{1}}(2,1) \psi\left(s_{1}\right) .
$$

Here $U_{s_{i} s_{i-1}}(i, i-1)$ is the amplitude to go from $s_{i-1}$ at $t=i-1$ to $s_{i}$ at $t=i$ and $\psi\left(s_{1}\right)=<e_{s_{1}}, \psi>$. The decoherence functional on the full event algebra $\mathfrak{A}$ is then obtained using its bi-additivity property.

The restricted evolution from the initial state $\psi \in \mathcal{H}_{1}$ with respect to a truncated history $\gamma$ is defined to be

$$
\widetilde{\psi}_{\gamma} \equiv \widehat{C}_{\gamma} \psi \in \mathcal{H}_{m},
$$

where the class operator

$$
\widehat{C}_{\gamma} \equiv P_{s_{m}} U(m, m-1) P_{s_{m-1}} U(m-1, m-2) \ldots U(2,1) P_{s_{1}}
$$

and $P_{s}$ is the projector that projects onto the basis state $e_{s}$. Evolving $\widetilde{\psi}_{\gamma}$ back to the initial time gives us the state

$$
\psi_{\gamma} \equiv(U(m, m-1) U(m-1, m-2) \ldots U(2,1))^{\dagger} \widehat{C}_{\gamma} \psi \in \mathcal{H}_{1},
$$

and it can be shown that the decoherence functional

$$
D\left(\operatorname{cyl}(\gamma), \operatorname{cyl}\left(\gamma^{\prime}\right)\right) \equiv\left\langle\psi_{\gamma}, \psi_{\gamma^{\prime}}\right\rangle .
$$

For any $\alpha \in \mathfrak{A}$ and a partition into cylinder sets $\alpha=\cup_{i=1}^{k} \operatorname{cyl}\left(\gamma_{k}\right)$, one can also define the state

$$
\psi_{\alpha}=\sum_{k} \psi_{\gamma_{k}}
$$

and show that

$$
\left\langle\psi_{\alpha}, \psi_{\beta}\right\rangle=D(\alpha, \beta) .
$$

In 9 it was shown that for a finite $N$ dimensional system which evolves unitarily with discrete time steps there is, generically, an explicit, physically meaningful isomorphism $f: \mathcal{H} \rightarrow \mathcal{H}_{1}$ between the histories Hilbert space $\mathcal{H}$ and the standard Hilbert space $\mathcal{H}_{1}=\mathbb{C}^{N}$. Namely,

$$
f\left(\left[\left\{u_{i}\right\}\right]\right) \equiv \lim _{i \rightarrow \infty} f_{0}\left(u_{i}\right),
$$

with $f_{0}: V \rightarrow \mathcal{H}_{0}$ given by

$$
f_{0}(u) \equiv \sum_{\alpha \in \mathfrak{A}} u(\alpha) \psi_{\alpha} .
$$


Here $\left\langle f_{0}(u), f_{0}(v)\right\rangle=\langle u, v\rangle$ and hence $\left\langle f\left(\left[\left\{u_{i}\right\}\right]\right), f\left(\left[\left\{v_{i}\right\}\right]\right)\right\rangle=\left\langle\left[\left\{u_{i}\right\}\right],\left[\left\{v_{i}\right\}\right]\right\rangle$. The quantum vector pre-measure $\mu_{\mathrm{v}}: \mathfrak{A} \rightarrow \mathcal{H}$ is therefore mapped to a vector measure $\tilde{\mu_{\mathrm{v}}} \equiv f \circ \mu_{\mathrm{v}}$ on $\mathcal{H}_{1}$

$$
\begin{aligned}
\tilde{\mu}_{\mathrm{v}}(\alpha) & =\sum_{\beta \in \mathfrak{A}} \chi_{\alpha}(\beta) \psi_{\beta} \\
& =\psi_{\alpha} .
\end{aligned}
$$

Claim 3 The quantum vector measure for a generic (to be defined) finite unitary system with discrete time steps is not of bounded variation.

Proof: In what follows we will first assume that $U$ is time independent so that $U(k+1, k)=U$ for all $k$, where $U$ is the single time-step evolution operator.

For a string of length $m, \gamma=\left(j_{1}, j_{2}, \ldots j_{m}\right)$

$$
\tilde{\mu_{\mathrm{v}}}(\operatorname{cyl}(\gamma))=\left(U^{\dagger}\right)^{m} U_{j_{m} j_{m-1}} U_{j_{m-1} j_{m-2}} \ldots U_{j_{2} j_{1}} \beta_{j_{1}} e_{j_{1}}
$$

where $U_{j k}=\left\langle e_{j}, U e_{k}\right\rangle$ and $\beta_{j_{1}}=\left\langle e_{j_{1}}, \psi\right\rangle$, so that

$$
\left\|\tilde{\mu_{\mathrm{v}}}(\operatorname{cyl}(\gamma))\right\|=\left|\beta_{j_{1}}\right|\left|U_{j_{m} j_{m-1}} U_{j_{m-1} j_{m-2}} \ldots U_{j_{2} j_{1}}\right| .
$$

A string $\gamma^{\prime}$ of length $m+k$ will be said to be an extension of $\gamma$ if the first $m$ entries of $\gamma^{\prime}$ are the same, so that $\operatorname{cyl}\left(\gamma^{\prime}\right) \subset \operatorname{cyl}(\gamma)$. The set of all $n+k$ extensions $\left\{\operatorname{cyl}\left(\gamma^{\left(i_{1} i_{2} \ldots i_{k}\right)}\right)\right\}$ of $\gamma$ thus provides a partition of $\operatorname{cyl}(\gamma)$ so that

$$
\begin{aligned}
\left|\tilde{\mu}_{\mathrm{v}}\right|(\operatorname{cyl}(\gamma)) \geq & \sum_{i_{1}, i_{2} \ldots i_{k}=1}^{N} \| \tilde{\mu_{\mathrm{v}}}\left(\operatorname{cyl}\left(\gamma^{\left(i_{1} i_{2} \ldots i_{k}\right)}\right) \|\right. \\
= & \left(\sum_{\substack{i_{1}, i_{2} \ldots, i_{k}=1\\
}}^{N}\left|U_{i_{k} i_{k-1}}\right|\left|U_{i_{k-1} i_{k-2}}\right| \ldots\left|U_{i_{1} j_{m}}\right|\right) \\
& \times\left\|\tilde{\mu}_{\mathrm{v}}(\operatorname{cyl}(\gamma))\right\| .
\end{aligned}
$$

Since $U$ is unitary, $\sum_{i=1}^{N}\left|U_{i j}\right|^{2}=1$ and $\sum_{j=1}^{N}\left|U_{i j}\right|^{2}=1$. Thus,

$$
\begin{aligned}
& \sum_{i=1}^{N}\left|U_{i j}\right|=1+\zeta_{j} \\
& \sum_{j=1}^{N}\left|U_{i j}\right|=1+\eta_{i}
\end{aligned}
$$

where $\zeta_{j} \geq 0$ and $\eta_{i} \geq 0$. If $\zeta_{j}=0$ then there exists an $i$ such that $\left|U_{i j}\right|=1$ and $U_{i^{\prime} j}=0$ for all $i^{\prime} \neq i$. Similarly, for every $j^{\prime} \neq j, U_{i j^{\prime}}=0$. Hence the $i$ th row and the $j$ th column each have a single non-zero entry $U_{i j}$ which is pure phase.

We now define the genericity assumption for the time-independent case to be that $U$ does not have such entries, i.e., $\zeta_{j}>0$ for all $j$, and hence $\eta_{i}>0$ for all $i$. This excludes dynamics which consist of simple permutations of a number of 
the site. This assumption means that there is a smallest strictly positive $\zeta \leq \zeta_{j}$ for all $j$. This allows us to iteratively bound the term in brackets in (35)

$$
\begin{aligned}
\sum_{i_{k-1}, \ldots i_{1}=1}^{N}\left(\sum_{i_{k}}\left|U_{i_{k} i_{k-1}}\right|\right) & \times\left|U_{i_{k-1} i_{k-2}}\right| \ldots\left|U_{i_{1} j_{m}}\right| \\
& =\sum_{i_{k-2}, \ldots i_{1}=1}^{N}\left(\sum_{i_{k-1}=1}^{N}\left(1+\zeta_{i_{k-1}}\right)\left|U_{i_{k-1} i_{k-2}}\right|\right) \ldots\left|U_{i_{1} j_{m}}\right| \\
& \geq(1+\zeta) \sum_{i_{k-2}, \ldots i_{1}=1}^{N}\left(\sum_{i_{k-1}=1}^{N}\left|U_{i_{k-1} i_{k-2}}\right|\right) \ldots\left|U_{i_{1} j_{m}}\right| \\
& \geq(1+\zeta)^{k}
\end{aligned}
$$

Hence for every $k$,

$$
\left|\tilde{\mu_{\mathrm{v}}}\right|(\operatorname{cyl}(\gamma)) \geq(1+\zeta)^{k} \times\left\|\tilde{\mu}_{\mathrm{v}}(\operatorname{cyl}(\gamma))\right\|
$$

Since $\zeta>0,\left|\tilde{\mu_{\mathrm{v}}}\right|$ is not bounded.

For time dependent $U$ the argument is similar, except that the $\zeta$ are now time dependent. In particular, instead of extracting a time-independent factor $(1+\zeta)$ in Eqn (38) at every step, it becomes time-dependent so that

$$
\left|\tilde{\mu}_{\mathrm{v}}\right|(\operatorname{cyl}(\gamma)) \geq \prod_{r=1}^{m}\left(1+\zeta_{r}\right)\left\|\tilde{\mu}_{\mathrm{v}}(\operatorname{cyl}(\gamma))\right\|
$$

where $\sum_{i=1}^{N}\left|U_{i j}(r, r-1)\right|=1+\zeta_{j}(r)$ and $\zeta_{r}$ is the lowest value of $\zeta_{j}(r)$ as one varies over $j$. Again, assuming that $\zeta_{r}>0$, the product $\prod_{r=1}^{m}\left(1+\zeta_{r}\right)$ converges as $m \rightarrow \infty$ only if $\sum_{r=1}^{m} \zeta_{r}$ does. The genericity condition is therefore that $\sum_{r=1}^{m} \zeta_{r}$ diverges as $m \rightarrow \infty$ in which case (40) diverges and the pre-measure is not of bounded variation.

Thus the quantum vector pre-measure $\tilde{\mu}_{\mathrm{v}}$ on $\mathfrak{A}$ does not extend to a quantum vector measure on $\mathfrak{S}_{\mathfrak{A}}$ for generic finite dimensional unitary systems with discrete time steps.

\section{Complex Percolation}

In causal set theory, the histories space of continuum spacetime geometries is replaced by the collection of causal sets. A causal set $C$, as defined in [13], is a locally finite partially ordered set, namely, a countable collection of elements, with an order relation $\prec$ which for all $x, y, z \in C$ is (i) transitive $(x \prec y, y \prec$ $z \Rightarrow x \prec z$ ), (ii) irreflexive, $(x \nprec x)$ and (iii) locally finite i.e. if $\operatorname{Past}(x) \equiv$ $\{w \in C \mid w \prec x\}$ and $\operatorname{Fut}(x) \equiv\{w \in C \mid w \succ x\}$ then the cardinality of the set Past $(x) \cap F u t(y)$ is finite. We say that two elements are linked if they are related in the order but there is no element between them in the order. 
A causal set is a model for discrete spacetime in which the elements of $C$ represent spacetime events and the partial order represents the causal relationships between events. A generic causal set has no continuum spacetime approximation, however, and it is only via an appropriate choice of dynamics on the set of all possible causal sets that one expects a continuum spacetime to emerge.

The transitive percolation dynamics for causal sets is a classical stochastic dynamics and was studied in detail in 14, 15, and is determined by a single coupling constant $p \in[0,1]$. Here, a causal set is "grown" element by element starting with a single element. At stage $n>1$ the $n$th element $e_{n}$ is born and, for each $k=1,2 \ldots n-1$ independently, $e_{n}$ is put to the future of $e_{k}$ with probability $p$ or with probability $1-p$ left unrelated to $e_{k}$. The transitive closure is then taken and the stage $n$ is complete. The resulting causal set grown in this way is "labelled" by the growth, with each element labelled by the stage at which it is born (see Fig 1). Thus, the growth process is stochastic and produces a labelled causal set of infinite cardinality in the asymptotic limit $n \rightarrow \infty$. Such a causal set is always past finite, i.e., the cardinality of the past set $\operatorname{Past}(x)=\{y \mid y \prec x\}$ is finite for all $x \in C$. Even though the causal sets produced are labelled, the resultant dynamics satisfies a discrete form of general covariance in that the probabilities of growing, by stage $n$, two labelled causal sets are the same if there is an order preserving isomorphism between them. In addition, the dynamics satisfies the "Bell causality" condition described in [14. The labelling of a causal set produced via a growth is always order preserving namely, for any $e, e^{\prime} \in C^{n}, e \prec e^{\prime}$ implies that $l(e)<l\left(e^{\prime}\right)$, where $l(e)$ is the label of the element $e$.

Figure 1 show the first few stages of this growth process. Let $C_{c}^{n}$ denote the $n$-chain or the totally ordered set of $n$ elements and $C_{a}^{n}$ the $n$-antichain or the set of $n$ mutually unrelated elements. Starting with the first element $e_{1}$ the second element $e_{2}$ is added with probability $p \in[0,1]$ to the future of $e_{1}$, to get the (uniquely) labelled 2-chain $C_{c}^{2}$ or is left unrelated to $e_{1}$ with probability $q$ to get the (uniquely) labelled 2-antichain $C_{a}^{2}$. Since for $n=2$ these are the only possible 2 element labelled causal sets, $p+q=1$ (see Fig 1). Subsequently, the three 3-element causal sets are grown from $C_{c}^{2}$ and $C_{a}^{2}$ as shown in Fig 1 In the first transition from $C_{c}^{2}$ in the figure (from the left), $e_{3}$ is added to the immediate future of $e_{2}$ with probability $p$, in the second, $e_{3}$ is added to the immediate future of $e_{1}$ but is unrelated to $e_{2}$ with probability $p \times q$, and in the third, $e_{3}$ is unrelated to both $e_{1}$ and $e_{2}$ with probability $q^{2}$. In the figure we see that the middle three 3 -element labelled causal sets are different order preserving relabellings of the same unlabelled causal set.

The probability $P\left(C^{n}\right)$ for an $n$-element labelled causal set $C^{n}$ is equal to the product of the transition probabilities. As can be verified from the examples in Fig 1 this probability is independent of the labelling: in general, the probability for a labelled causal set is given by $p^{L} q^{\left(\begin{array}{c}n \\ 2\end{array}\right)-R}$ where $L$ is the number of links and $R$ the number of relations [14. The transition probability for going from an $n$-element causal set $C^{n}$ - a parent - to one of its children $C^{n+1}$ is given by a product of $p$ 's and $q$ 's: if the new element is to the immediate future of (i.e. 


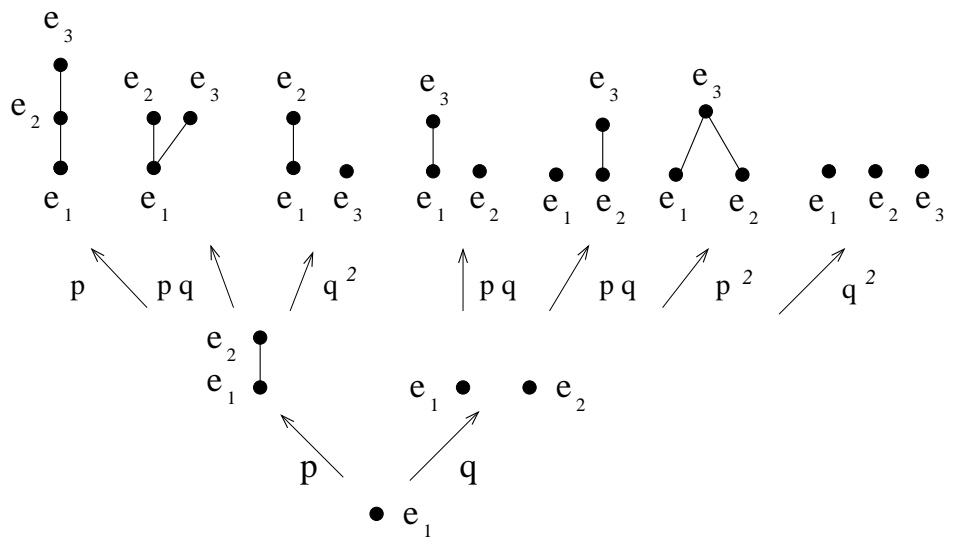

Figure 1: Transition probabilities up to 3-element labelled causal sets.

linked to) u elements in $C^{n}$ and unrelated to v elements, then the transition amplitude is $p^{\mathrm{u}} q^{\mathrm{v}}$. For a given parent $C^{n}$ therefore, one can assign an index set $\mathcal{I}\left(C^{n}\right)$ of all pairs $(\mathrm{u}, \mathrm{v})$, some of which may repeat. For example, when the parent is $C_{c}^{2}$, the index set is $\mathcal{I}\left(C_{c}^{2}\right)=\{(1,0),(1,1),(0,2)\}$ while if the parent is $C_{a}^{2}$, the index set is $\mathcal{I}\left(C_{a}^{2}\right)=\{(1,0),(1,0),(2,0),(0,2)\}$ (see Fig 1). Note that the set $\mathcal{I}\left(C^{n}\right)$ can contain repeated entries.

It is useful to point to two special types of transitions which will make their appearance in our analysis below. The first is the "timid transition" in which the new element is added to the future of all the elements in $C^{n}$, so that the transition probability is $p^{m}$ where $m$ is the number of maximal elements in $C^{n}$. Thus, $(\mathrm{u}, \mathrm{v})=(m, 0)$. The second is the "gregarious transition" in which the new element is unrelated to all the existing elements in $C^{n}$, so that the transition probability is $q^{n}$ and $(\mathrm{u}, \mathrm{v})=(0, n)$.

Let $\Omega$ be the set of all infinite, past finite, labelled causal sets. If $C^{n}(k)$ refers to the $k$ th labelled element in $C^{n}$, the cylinder set associated with $C^{n}$ is

$$
\operatorname{cyl}\left(C^{n}\right) \equiv\left\{c \in \Omega \mid c(k)=C^{n}(k), k=1, \ldots, n\right\}
$$

where $c(k)$ is the $k$ th element of $c$. The event algebra $\mathfrak{A}$ is the set of all finite unions of these cylinder sets and it can be shown that every element of $\mathfrak{A}$ is equal to a finite union of mutually disjoint cylinder sets. In particular $\operatorname{cyl}\left(C^{n}\right)$ is equal to the union of the (disjoint) cylinder sets of all the children of $C^{n}$ : $\operatorname{cyl}\left(C^{n}\right)=\bigcup_{i} \operatorname{cyl}\left(C_{i}^{n+1}\right)$. However, unlike the finite unitary systems, the number of $n+1$-element children $\left\{C_{i}^{n+1}\right\}$ depends on the parent $C^{n}$.

Complex percolation is a natural quantum generalisation of transitive percolation in which real probabilities are replaced by complex amplitudes. Thus, the real parameter $p$ of transitive percolation is made complex and gives the transition amplitude for the newly born element to be put to the future of each existing element, while $q$ is the transition amplitude for it to be unrelated (we 
will see later that $p+q=1$ ). The decoherence functional for complex percolation has a simple product form

$$
D\left(\operatorname{cyl}\left(C^{n}\right), \operatorname{cyl}\left(C^{\prime n}\right)\right)=A^{*}\left(C^{n}\right) A\left(C^{\prime n}\right)
$$

for cylinder sets, where $A\left(C^{n}\right)$ is the amplitude for the transition from the empty set to the $n$-element causal set $C^{n}$. Finite biadditivity of $D$ and finite additivity of $A$ are equivalent and $A$ is a complex measure on $\mathfrak{A}$. The normalisation condition $D(\Omega, \Omega)=1$ implies that $|A(\Omega)|=1$. We have for $\alpha, \beta \in \mathfrak{A}$,

$$
D(\alpha, \beta)=A^{*}(\alpha) A(\beta) .
$$

It is easy to demonstrate that for any such "product" decoherence functional the histories Hilbert space $\mathcal{H} \simeq \mathbb{C}$. Choose a vector $v \in V$, with $\|v\| \neq 0$. We show that for every $u \in V$ there exists a $\lambda \in \mathbb{C}$ such that $\{u\} \sim \lambda\{v\}$, where $\sim$ is the equivalence relation Eqn (5) and $\{u\},\{v\}$ are the constant Cauchy sequences for $u$ and $v$ respectively. Then

$$
\|u-\lambda v\|^{2}=\left|S_{1}\right|^{2}-\left(\lambda S_{1}^{*} S_{2}+\lambda^{*} S_{1} S_{2}^{*}\right)+|\lambda|^{2}\left|S_{2}\right|^{2}=\left|S_{1}-\lambda S_{2}\right|^{2},
$$

where $S_{1}=\sum_{\alpha \in \mathfrak{A}} A(\alpha) u(\alpha)$ and $S_{2}=\sum_{\alpha \in \mathfrak{A}} A(\alpha) v(\alpha)$. This factorisation is possible because of the product form Eqn (43). If we then choose $\lambda=S_{1} / S_{2}$ $\left(S_{2} \neq 0\right)$ we have $\|u-\lambda v\|=0$.

In particular, since for any $\alpha \in \mathfrak{A}$ and a finite partition $\pi(\alpha)=\left\{\alpha_{\rho}\right\}$ of $\alpha$

$$
\left|\mu_{\mathrm{v}}\right|(\alpha) \geq \sum_{\rho}\left\|\mu_{\mathrm{v}}\left(\alpha_{\rho}\right)\right\|=\sum_{\rho}\left|A\left(\alpha_{\rho}\right)\right|
$$

Hence $\left|\mu_{\mathrm{v}}\right|=|A|$, where $|A|$ the total variation of $A$, and $\mu_{\mathrm{v}}$ is equal to $A$ up to a phase.

For the special case $p$ real and $p \in[0,1]$, the transition amplitudes are the same as the transition probabilities of classical transitive percolation. However, this real quantum percolation model is distinct from transitive percolation since the quantum measure $D(\alpha, \alpha)=|A(\alpha)|^{2}$ and is therefore nonadditive. However, the amplitude measure $A$ is additive and non-negative, and the Carathéodory-Kolmogorov extension theorem implies that the quantum vector pre-measure extends to the full-sigma algebra. As we will presently see, this special case is the only one which does admit such an extension.

We first show that $q$ must equal $1-p$. The normalisation condition on $D$ means that $|A(\Omega)|=1$, so that $A(\Omega)=\exp (i \Phi)$ and we will choose this phase to be 1 . If $C^{1}$ denotes the single element causal set then $\operatorname{cyl}\left(C^{1}\right)=\Omega$. We also have $\operatorname{cyl}\left(C^{1}\right)=\operatorname{cyl}\left(C_{c}^{2}\right) \cup \operatorname{cyl}\left(C_{a}^{2}\right)$ and $\operatorname{cyl}\left(C_{c}^{2}\right) \cap \operatorname{cyl}\left(C_{a}^{2}\right)=\varnothing$, where, as before $C_{c}^{2}$ and $C_{a}^{2}$ are the 2-chain and 2-antichain respectively. This means

$$
\begin{aligned}
A\left(\operatorname{cyl}\left(C^{1}\right)\right) & =A\left(\operatorname{cyl}\left(C_{c}\right)\right)+A\left(\operatorname{cyl}\left(C_{a}\right)\right) \\
& =A\left(\operatorname{cyl}\left(C^{1}\right)\right) \times(p+q) \Rightarrow p+q=1 .
\end{aligned}
$$

As shown above, the histories Hilbert space for a product decoherence functional is 1-dimensional. This means that the quantum vector measure is, up to an overall phase, just the amplitude $A: \mathfrak{A} \rightarrow \mathbb{C}$.

We now show that 

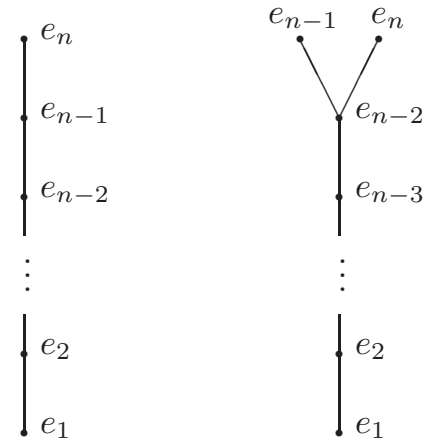

Figure 2: Hasse diagrams for $C_{c}^{n}$ and $C_{v}^{n}$.

Lemma 1 The quantum vector measure of complex percolation is not of bounded variation when the parameter $p$ is not real.

We begin by considering the set of all labelled $n$-element causets $\left\{C_{i}^{n}\right\}$, where $i=1,2, \ldots I(n)$ where $I(n)$ is the number of $n$ element labelled causal sets. For example, for $n=2, I(2)=2$ so that $i=1,2$ and for $n=3, I(3)=7$, so that $i=1, \ldots, 7$. Since $A(\Omega)=\sum_{i=1}^{I(n)} A\left(\operatorname{cyl}\left(C_{i}^{n}\right)\right)$, and $|A(\Omega)|=1$, the triangle inequality implies that

$$
\sum_{i=1}^{I(n)}\left|A\left(C_{i}^{n}\right)\right| \geq 1
$$

where for brevity of notation we have replaced $\operatorname{cyl}\left(C^{n}\right)$ with $C^{n}$. The equality is satisfied only if all the $A\left(C_{i}^{n}\right)$ are collinear. We see that

Claim 4 For any $n \geq 2$, the equality in (46) is satisfied only when the parameter $p$ is real.

Proof: Let $p=|p| \exp ^{i \theta}, q=|q| \exp ^{i \phi}$. First, for $n=2$ the equality means that $|p|+|q|=1$, which combined with $p+q=1$ means that $p$ is real and non-negative. For $n>2$, consider the following two $n$ element causal sets, (a) the $n$ chain $C_{c}^{n}$ and (b) $C_{v}^{n}$, an $n-2$ chain topped with a " $V$ ", i.e. with $e_{n}$, $e_{n-1}$ to the immediate future of the maximal element $e_{n-2}$ of the $n-2$ chain $C_{c}^{n-2}$, and unrelated to each other (see Fig 2). The amplitudes for these causal sets are $A\left(C_{c}^{n}\right)=p^{n-1}$, and $A\left(C_{v}^{n}\right)=p^{n-1} q$. Requiring collinearity of these amplitudes is therefore equivalent to requiring that $p$ is real and non-negative.

Thus, for $p$ non-real

$$
\sum_{i=1}^{I(n)}\left|A\left(C_{i}^{n}\right)\right|>1 .
$$


for all $n \geq 2$. For $n=2$ it is useful to express the inequality as

$$
|p|+|q|=1+\zeta, \zeta>0
$$

Claim 5 If $|p|>1$ or $|q|>1$, the quantum vector measure is not of bounded variation.

Proof: Consider any partition of $\Omega$ which contains $\operatorname{cyl}\left(C_{c}^{n}\right)$. Since $A\left(C_{c}^{n}\right)=$ $p^{n-1},|A|(\Omega)>|p|^{n-1}$ is a strict inequality for any $n$ if $q \neq 0$. Similarly, consider any partition of $\Omega$ which contains $\operatorname{cyl}\left(C_{a}^{n}\right)$. Since $A\left(C_{a}^{n}\right)=q^{n(n-1)}$, $|A|(\Omega)>|q|^{n(n-1)}$ for any $n$, if $p \neq 0$. Thus, $A$ is not of bounded variation if either $|p|>1$ or $|q|>1$.

Thus, we may restrict our attention to $|p|<1,|q|<1$. Consider an nelement causal set $C^{n}$. Let $\left\{C_{j_{1}}^{n+1}\right\}$ be the set of its $n+1$ element descendants and $a\left(j_{1}\right)$ the associated transition amplitude. The index $j_{1}=1,2, \ldots J_{1}\left(C^{n}\right)$ where $J_{1}\left(C^{n}\right)$ are the number of descendants of $C^{n}$. In turn, let $C_{j_{1} j_{2}}^{n+2}$ denote the $n+2$ element descendant of $C_{j_{1}}^{n+1}$ and $a_{j_{1}}\left(j_{2}\right)$ the associated transition amplitude, and so on. The index $j_{2}$ depends on $j_{1}$ since $j_{2}=1,2, \ldots J_{2}\left(C_{j_{1}}^{n}\right)$ where $J_{2}\left(C_{j_{1}}^{n}\right)$ are the number of descendants of $C_{j_{1}}^{n+1}$ and so on. $j_{2}$ thus carries a hidden index $j_{1}$, but we will not include it explicitly in the expressions below. The set $\Pi \equiv\left\{C_{j_{1} j_{2} \ldots j_{s}}^{n+s}\right\}$ of $n+s$ element descendants of $C^{n}$ provides a disjoint partition of $\operatorname{cyl}\left(C^{n}\right)$ where the range $J_{r}\left(C_{j_{1} j_{2} \ldots j_{r-1}}^{n+r-1}\right)$ of each $j_{r}$ is determined by its parent $C_{j_{1} j_{2} \ldots j_{r-1}}^{n+r-1}$. Thus the total variation

$$
|A|\left(C^{n}\right) \geq \sum_{j_{1}=1}^{J_{1}}\left(\sum_{j_{2}=1}^{J_{2}\left(j_{1}\right)}\left(\ldots\left(\sum_{j_{s}=1}^{J_{s}\left(j_{s_{1}} j_{s-2} \ldots j_{1}\right)}\left|A\left(C_{j_{1} j_{2} \ldots j_{s}}^{n+s}\right)\right|\right) \ldots\right)\right)
$$

where

$$
A\left(C_{j_{1} j_{2} \ldots j_{s}}^{n+s}\right)=A\left(C^{n}\right) \times a\left(j_{1}\right) a_{j_{1}}\left(j_{2}\right) \ldots a_{j_{s-1}}\left(j_{s}\right)
$$

Thus

$$
\begin{array}{r}
|A|\left(C^{n}\right) \geq\left|A\left(C^{n}\right)\right| \times\left(\sum _ { j _ { 1 } } | a ( j _ { 1 } ) | \left(\sum_{j_{2}}\left|a_{j_{1}}\left(j_{2}\right)\right|(\ldots\right.\right. \\
\left.\left.\left.\left(\sum_{j_{s}}\left|a_{j_{s-1}}\left(j_{s}\right)\right|\right) \ldots\right)\right)\right),
\end{array}
$$

where we have suppressed the dependencies of the $j_{r}$ 's. We now show that

$$
\sum_{j_{i}}\left|a_{j_{i-1}}\left(j_{i}\right)\right| \geq 1+\zeta
$$

for every $i$. 
The final sum within the nested brackets of (51)

$$
\sum_{j_{s}}\left|a_{j_{s-1}}\left(j_{s}\right)\right| \geq 1
$$

since

$$
\sum_{j_{s}} a_{j_{s-1}}\left(j_{s}\right)=1
$$

Now, as in transitive percolation, each term in (54) is of the form $p^{\mathrm{u}} q^{\mathrm{v}}$, with $(\mathrm{u}, \mathrm{v}) \in \mathcal{I}$, where we have suppressed the dependence of the index set $\mathcal{I}$ on the parent $C_{j_{1} j_{2} \ldots j_{s-1}}^{n+s-1}$. If $m$ is the maximal number of elements in $C_{j_{1} j_{2} \ldots j_{s-1}}^{n+s-1}$, and $\mathcal{I}^{\prime}$ is the index set which excludes $(\mathrm{u}, \mathrm{v})=(m, 0)$, then

$$
\begin{aligned}
\sum_{j_{s}} a_{j_{s-1}}\left(j_{s}\right) & =\sum_{(\mathrm{u}, \mathrm{v}) \in \mathcal{I}} p^{\mathrm{u}} q^{\mathrm{v}} \\
& =p^{m}+\sum_{(\mathrm{u}, \mathrm{v}) \in \mathcal{I}^{\prime}} p^{\mathrm{u}} q^{\mathrm{v}} \\
& =1+\sum_{\mathrm{w} \in W} c_{\mathrm{w}} q^{\mathrm{w}},
\end{aligned}
$$

for some appropriate index set $W$ and coefficients $c_{\mathrm{w}}$. Since the above sum is always equal to 1 , and is true for all $q$, this means that $c_{\mathrm{w}}=0$. Thus,

$$
\begin{aligned}
\sum_{j_{s}}\left|a_{j_{s-1}}\left(j_{s}\right)\right|= & |p|^{m}+\sum_{(\mathrm{u}, \mathrm{v}) \in \mathcal{I}^{\prime}}|p|^{\mathrm{u}}|q|^{\mathrm{v}} \\
= & (1+\zeta-|q|)^{m}+\sum_{i}(1+\zeta-|q|)^{u_{i}}|q|^{v_{i}} \\
= & (1-|q|)^{m}+\sum_{(\mathrm{u}, \mathrm{v}) \in \mathcal{I}^{\prime}}(1-|q|)^{\mathrm{u}}|q|^{\mathrm{v}}+ \\
& \left(\begin{array}{c}
m \\
1
\end{array}\right)(1-|q|)^{m-1} \zeta+\left(\begin{array}{c}
m \\
2
\end{array}\right)(1-|q|)^{m-2} \zeta^{2} \ldots+\zeta^{m}+ \\
& \sum_{(\mathrm{u}, \mathrm{v}) \in \mathcal{I}^{\prime \prime}}\left(\begin{array}{c}
\mathrm{u} \\
1
\end{array}\right)(1-|q|)^{\mathrm{u}-1} \zeta+\left(\begin{array}{c}
\mathrm{u} \\
2
\end{array}\right)(1-|q|)^{\mathrm{u}-2} \zeta^{2}+ \\
& \left.\ldots+\zeta^{\mathrm{u}}\right)|q|^{\mathrm{v}},
\end{aligned}
$$

where $\mathcal{I}^{\prime \prime}$ is the index set which excludes both $(\mathrm{u}, \mathrm{v})=(m, 0)$ and $(\mathrm{u}, \mathrm{v})=$ $(0, n+s-1)$. The first two terms are of the same form as expression (55) and hence equal to 1 since it is independent of the choice of $|q|$. Since $0 \leq|q| \leq 1$, each of the terms in the above expression is positive. It therefore suffices to 
focus on the terms linear in $\zeta$. We see that this can be simplified to

$$
\begin{aligned}
& \left((1-|q|)^{m}+\sum_{(\mathrm{u}, \mathrm{v}) \in \mathcal{I}^{\prime \prime}}(1-|q|)^{\mathrm{u}}|q|^{\mathrm{v}}\right)(1-|q|)^{-1} \\
& +\left((m-1)(1-|q|)^{m-1}+\sum_{(\mathrm{u}, \mathrm{v}) \in \mathcal{I}^{\prime \prime}}\left((\mathrm{u}-1)(1-|q|)^{\mathrm{u}-1}|q|^{\mathrm{v}}\right)\right) \\
> & \left.\left(1-|q|^{n+s-1}\right)\right)(1-|q|)^{-1} .
\end{aligned}
$$

For any $n+s-1 \geq 1$ the above expression is $>1$ for $|q|<1$. Thus, each nested sum $\sum_{j_{s}}\left|a_{j_{s-1}}\left(j_{s}\right)\right| \geq(1+\zeta)$, for any $j_{s-1}$ and hence from (51) we see that

$$
|A|\left(C^{n}\right) \geq\left|A\left(C^{n}\right)\right| \times(1+\zeta)^{s} .
$$

Since $s$ can be made arbitrarily large, this means the $|A|\left(C^{n}\right)$ is not bounded.

\section{Discussion}

We have seen that for a class of finite dimensional systems the quantum vector pre-measure on $\mathfrak{A}$ does not in fact admit extensions to the sigma algebra $\mathfrak{S}_{\mathfrak{A}}$ generated by $\mathfrak{A}$. We now discuss the implications of these results.

To start with, the lack of an extension does not by itself imply that no physical observables can be constructed. For the finite unitary systems all the events in $\mathfrak{A}$ are measurable and hence are physical observables. The lack of an extension simply means that while finite time questions are observables, not all infinite time questions are.

For causal sets on the other hand, one is interested in covariant or label invariant observables. Since the growth process generates only labelled causal sets, the events in $\mathfrak{A}$ are not themselves observables. One method of constructing covariant events [16] is to first generate the labelled sigma algebra $\mathfrak{S}_{\mathfrak{A}}$ and then take its quotient $\mathfrak{S}^{\prime}$ with respect to relabellings. $\mathfrak{S}^{\prime}$ is a sigma algebra over the space of unlabelled past-finite causal sets $\Omega^{\prime}$, and is collection of covariant events. For the classical stochastic growth models of [14 since the extension of the probability pre-measure to $\mathfrak{S}_{\mathfrak{A}}$ is guaranteed starting from a given probability pre-measure on $\mathfrak{A}$ this procedure gives rise to a unique covariant probability measure space $\left(\Omega^{\prime}, \mathfrak{S}^{\prime}, \mu^{\prime}\right)$, where $\mu^{\prime}$ is a covariant measure. If a quantum vector pre-measure extends to a vector measure on $\mathfrak{S}_{\mathfrak{A}}$, a similar procedure will give rise to a collection of covariant quantum observables.

When $p$ is real, i.e., for real quantum percolation, the pre-measure does extend to $\mathfrak{S}_{\mathfrak{A}}$, and hence covariant quantum observables can be constructed along the lines of [16. In particular, from the product form of the decoherence functional (Eqn (43)) it is trivial to see that all the covariant observables of classical transitive percolation are also observables for real quantum percolation.

In [16] it was shown that the covariantly defined and physically accessible stem 
sets generate a sub-sigma algebra $\mathfrak{S}_{S}^{\prime} \subset \mathfrak{S}^{\prime}$. A stem $c$ in a causal set $C$ is a subcausal set of $C$ which contains its own past 3 . The stem set, stem $(c) \subset \Omega^{\prime}$ is then the set of (unlabelled) causal sets which contains a stem isomorphic to $c$. Since the sets of measure zero in transitive percolation are also sets of measure zero in real quantum percolation, the results of [16] imply that for $p>0, \mathfrak{S}_{S}^{\prime}$ generates $\mathfrak{S}^{\prime}$ upto sets of measure zero. Thus, at least in this simple example of quantum dynamics, one recovers a complete set of covariant quantum observables.

As we have seen, for complex percolation with $p$ not real, $\mu_{v}$ does not extend to $\mathfrak{S}_{\mathfrak{A}}$, and hence the construction of covariant observables, if at all possible, requires a different approach. As discussed in Section 2 countable additivity of the measure requires an unconditional convergence of the right hand side of Eqn (3). It is this that implies bounded variation for finite dimensional vector measures. One option then is to require that the measure on $\mathfrak{S}_{\mathfrak{A}}$ satisfy only a conditional convergence, with the conditionality determined by a preferred ordering of events in $\mathfrak{A}$. The "nested" structure of the cylinder sets (Eqns (20)), (21) suggests a natural ordering on $\mathfrak{A}$ in terms of cardinality. For example, for a countable disjoint union of cylinder sets, a conditional convergence can be easily defined with respect to such an ordering. However, in order for this prescription to work in general, it is important to ensure that such an ordering procedure is unique.

A simpler alternative is to use transitive percolation as a template for the quantum vector measure, by first calculating the probabilities for transitive percolation and then complexifying them. In other words, if $P(\alpha)$ is the probability for the event $\alpha \in \mathfrak{S}_{\mathfrak{A}}$ for transitive percolation, then one defines $A(\alpha)$ to be an appropriately complexified version. As a concrete example, consider the event $\alpha_{o} \in \mathfrak{S}_{\mathfrak{A}}$ corresponding to the existence of an element which is to the past of all other element: 4 . The probability of such an event for transitive percolation is given by the Euler function

$$
\phi(q)=\prod_{i=1}^{\infty}\left(1-q^{i}\right) .
$$

Since $\phi(q)$ is finite for all $q \in \mathbb{C},|q|<1$, we can define $D\left(\alpha_{0}, \alpha_{0}\right) \equiv|\phi(q)|^{2}$. Whether this gives rise to a genuine finitely bi-additive quantum measure on $\mathfrak{S}_{\mathfrak{A}}$ would depend on the detailed nature of the complexification procedure adopted.

If successful, such prescriptions, though seemingly ad-hoc, would be in keeping with the attitude one adopts in physics. Namely, the failure of a quantum measure to have a "mathematical extension" does not mean that it cannot have a "physical extension". This happens in physics all the time: even though one often comes across non-convergent expressions, we can make sense of them by applying a physically meaningful cutoff and using the limit as the cutoff is taken away to define the quantity. We can do the same thing here: define limits

\footnotetext{
${ }^{3}$ A stem set is a discrete version of a past set for which $S=J^{-}(S)$, where $J^{-}(x)$ denotes the set of events in the causal past of $x$ [17.

${ }^{4} \mathrm{~A}$ causal set with an element to the past of all other elements is referred to as "originary".
} 
only in a physically meaningful way. Even if quantities are only conditionally convergent these "conditions" are physically determined.

On the other hand, the failure of bounded variation could also have implications for physical predictions. In classical probability theory, we expect that a set of zero quantum measure is one which almost surely does not happen or is precluded. However, sets of zero quantum measure can contain sets of non-zero quantum measure [1, 5. Without going into the details of an interpretational framework (see [18, 19]) it is reasonable to assume that any set contained in a set of zero quantum measure is also precluded and hence almost surely will not happen.

We now show that bounded variation is sufficient to ensure that not all elements of $\mathfrak{A}$ are contained in a set of measure zero for a large class of systems, and hence are not precluded.

Claim 6 For an event algebra $\mathfrak{A}$ generated by cylinder sets constructed from finite length strings as in (19) and which satisfy (20) and (21), if $\mu_{\mathrm{v}}$ is of bounded variation, then not every $\alpha \in \mathfrak{A}$ can be contained in a set of measure zero.

Proof: Assume the contrary, i.e., that every $\alpha \in \mathfrak{A}, \alpha \subset \Omega$ is either of measure zero or contained in a set of measure zero. Let $\Gamma(n)$ denote the set of length $n$ strings. For any $\tilde{\gamma} \in \Gamma(n)$ define $\tilde{\Gamma} \equiv \Gamma(n) \backslash \tilde{\gamma}$ and a disjoint union of cylinder sets $\alpha_{\tilde{\gamma}} \equiv \bigcup_{\gamma \in \tilde{\Gamma}} \operatorname{cyl}(\gamma) \in \mathfrak{A}$. Then there exists a $\beta_{\tilde{\gamma}} \supseteq \alpha_{\tilde{\gamma}}$ which is of quantum measure zero, i.e., $\left\|\mu_{\mathrm{v}}\left(\beta_{\tilde{\gamma}}\right)\right\|=D\left(\beta_{\tilde{\gamma}}, \beta_{\tilde{\gamma}}\right)=0$. This means that $\mu_{\mathrm{v}}\left(\beta_{\tilde{\gamma}}\right)$ is a zero vector in $\mathcal{H}$, so that $\mu_{\mathrm{v}}(\Omega)=\mu_{\mathrm{v}}\left(\beta_{\tilde{\gamma}}^{c}\right)$ where $\beta_{\tilde{\gamma}}^{c} \subseteq \operatorname{cyl}(\tilde{\gamma})$, by the additivity of $\mu_{v}$. Thus, $\left\|\mu_{\mathrm{v}}\left(\beta_{\tilde{\gamma}}^{c}\right)\right\|=1$. Now consider the following partition of $\Omega$. For each $\tilde{\gamma} \in \Gamma(n)$, express $\operatorname{cyl}(\tilde{\gamma})$ as the disjoint union $\beta_{\tilde{\gamma}}^{c} \bigcup\left(\operatorname{cyl}(\tilde{\gamma}) \backslash \beta_{\tilde{\gamma}}^{c}\right)$, so that $\Omega$ can be expressed as the disjoint union

$$
\Omega=\bigcup_{\tilde{\gamma} \in \Gamma(n)} \beta_{\tilde{\gamma}}^{c} \bigcup\left(\operatorname{cyl}(\tilde{\gamma}) \backslash \beta_{\tilde{\gamma}}^{c}\right) .
$$

Then,

$$
\left|\mu_{\mathrm{v}}\right|(\Omega) \geq \sum_{\tilde{\gamma} \in \Gamma(n)}\left\|\mu_{\mathrm{v}}\left(\beta_{\tilde{\gamma}}^{c}\right)\right\|=2^{n}
$$

for each $n$ and is hence unbounded.

While this does not prove that bounded variation is a necessary condition, the following is an example which is not of bounded variation, and for which every set in $\mathfrak{A}$ is contained in a set of measure zero and hence is precluded. In other words, the only event in $\mathfrak{A}$ that does occur is $\Omega$ itself! This system belongs to the class of generic unitary systems studied in Section 3 and hence is not of bounded variation.

\footnotetext{
${ }^{5}$ The term "cylinder sets" is also used to describe the sets that generate the event algebra for continuous times, for example for Brownian motion. These sets do not satisfy 201 and (21).
} 
Claim 7 For a two state system whose dynamics is determined by the unitary operator

$$
U=\frac{1}{\sqrt{2}}\left(\begin{array}{ll}
1 & i \\
i & 1
\end{array}\right)
$$

every element in $\mathfrak{A}$ is contained in a set of measure zero, except for $\Omega$.

Proof:

For a two dimensional system, $s_{i} \in\{1,2\}$. If $e_{1}, e_{2}$ are the orthonormal basis vectors of $\mathcal{H}_{1}$, the trivial evolution of this basis (i.e., via the identity map) to time $t=m$ gives the orthonormal basis vectors $e_{1}(m), e_{2}(m)$ of $\mathcal{H}_{m}$. Wlog, let the initial state $\psi=e_{1} \in \mathcal{H}_{1}$. For $U$ given by Eqn (62) the restricted evolution $\widetilde{\psi}_{\gamma}$ for any $m$-length truncated history $\gamma=\left(s_{1}, s_{2}, \ldots, s_{m}\right)$ is

$$
\widetilde{\psi}_{\gamma}=\widehat{C}_{\gamma} \psi=\left(\frac{1}{\sqrt{2}}\right)^{m-1}(i)^{f} e_{s_{m}} \delta_{s_{1}, 1}
$$

where $f \in\{0,1, \ldots m-1\}$ denotes the number of "flips", i.e., transitions from 0 to 1 or 1 to 0 in $\gamma$. For example, if $\gamma=(0,1,0)$, then $f=2$. For this choice of initial state, $\widetilde{\psi}_{\gamma}$ is identically zero on all histories with $s_{1}=2$, and moreover for $f$ even, $e_{s_{m}}=e_{1}(m)$ while for $f$ odd, $e_{s_{m}}=e_{2}(m)$. Thus, truncated histories with the same $m, f$ values have the same $\widetilde{\psi}_{\gamma}$.

If $\Gamma(m)$ denotes the set of all $m$-length strings, then the unrestricted evolution $\widetilde{\psi}_{\Omega}$ from $\psi$ is

$$
\begin{aligned}
\widetilde{\psi}_{\Omega} & =\sum_{\gamma \in \Gamma(m)} \widetilde{\psi}_{\gamma}=\sum_{\gamma \in \Gamma(m)} \widehat{C}_{\gamma} \psi \\
& =a_{1}(m) e_{1}(m)+a_{2}(m) e_{2}(m) .
\end{aligned}
$$

Clearly $\widetilde{\psi}_{\Omega}$ is independent of $m$ and satisfies the normalisation $\left\langle\widetilde{\psi}_{\Omega}, \widetilde{\psi}_{\Omega}\right\rangle=$ 1. Contributions to $a_{1}(m)$ come only from truncated histories with even $f$ and those to $a_{2}(m)$ only from truncated histories with odd $f$. The number of strings with precisely $f$ flips is $\left(\begin{array}{c}m-1 \\ f\end{array}\right)$, and hence summing over even and odd $f$ respectively we find

$$
\begin{aligned}
& a_{1}(m)=\left(\frac{1}{\sqrt{2}}\right)^{m-1} \sum_{j=0}^{j_{\max }}(-)^{j}\left(\begin{array}{c}
m-1 \\
2 j
\end{array}\right) \\
& a_{2}(m)=i\left(\frac{1}{\sqrt{2}}\right)^{m-1} \sum_{k=0}^{k_{\max }}(-)^{k}\left(\begin{array}{c}
m-1 \\
2 k+1
\end{array}\right),
\end{aligned}
$$

where, for $m$ even $j_{\max }=k_{\max }=\frac{1}{2}(m-2)$ and for $m$ odd $j_{\max }=\frac{1}{2}(m-1)$ and $k_{\max }=\frac{1}{2}(m-3)$.

For $m=4 q+1$ the binomial expansion for $(1+i)^{4 q}$ gives

$$
\begin{aligned}
& a_{1}(4 q+1)=\left(\frac{1}{\sqrt{2}}\right)^{4 q}(-)^{q} 2^{2 q} \\
& a_{2}(4 q+1)=0 .
\end{aligned}
$$


Now, for every $\gamma \in \Gamma(4 q+1)$ whose first entry is $s_{1}=1$ and which has precisely $2 q$ flips, the last entry $s_{4 q+1}=1$. Hence the strings with $2 q$ flips contribute only to $a_{1}(4 q+1)$, each with an amplitude $\left(\frac{1}{\sqrt{2}}\right)^{4 q}(-)^{q}$. Moreover, the number of truncated histories with precisely $f=2 q \Gamma_{1} \subset \Gamma(4 q+1)$ is $\left(\begin{array}{c}4 q \\ 2 q\end{array}\right)$. Since $\left(\begin{array}{l}4 q \\ 2 q\end{array}\right)>2^{2 q}$, it is therefore possible to pick a subset $\Gamma_{2}$ of $\Gamma_{1}$ so that

$$
\widetilde{\psi}_{\Omega}=\sum_{\gamma \in \Gamma_{2}} \widehat{C}_{\gamma} \psi
$$

The complement of the set $\bigcup_{\gamma \in \Gamma_{2}} \operatorname{cyl}(\gamma)$ is therefore of measure zero.

We now show that every cylinder set $\operatorname{cyl}(\tilde{\gamma})$ with $\tilde{\gamma}=\left(s_{1}, s_{2}, \ldots, s_{\tilde{m}}\right)$ contains an event $\alpha$ such that its complement is of zero measure. Since every event except $\Omega$ is in the complement of some cylinder set, it also belongs to the complement of $\alpha$ which suffices to prove our result.

Let $\tilde{f}$ be the number of flips in $\tilde{\gamma}$. Any event which is a subset of $\operatorname{cyl}(\tilde{\gamma})$ shares the first $\tilde{m}$ entries with $\tilde{\gamma}$ and hence has at least $\tilde{f}$ flips. Choose a $q$ such that $2 q>\tilde{f}$ and let $\Gamma_{\tilde{\gamma}} \subset \operatorname{cyl}(\tilde{\gamma})$ be the set of truncated histories of length $4 q+1$ with precisely $2 q$ flips. The cardinality of this set is $\left(\begin{array}{c}4 q-\tilde{m} \\ 2 q-\tilde{f}\end{array}\right)$ and each truncated history in it contributes a factor of $\left(\frac{1}{\sqrt{2}}\right)^{4 q}(-)^{q}$ to $a_{1}(4 q+1)$. Since $\tilde{f} \in\{0,1, \ldots, \tilde{m}\}$, if $q \geq 3$

$$
\left(\begin{array}{c}
4 q-\tilde{m} \\
2 q-\tilde{f}
\end{array}\right) \geq\left(\begin{array}{c}
4 q-\tilde{m} \\
2 q-\tilde{m}
\end{array}\right)>\left(\begin{array}{c}
3 q \\
q
\end{array}\right) \geq 2^{2 q} .
$$

Thus, there exists a $\Gamma_{2} \subset \Gamma_{\tilde{\gamma}} \subset \Gamma_{1}$ for which Eqn (68) is satisfied so that the complement of the event $\bigcup_{\gamma \in \Gamma_{2}}$ is of measure zero. Thus, every event in the complement of $\operatorname{cyl}(\tilde{\gamma})$ is contained in a set of measure zero.

Since this is true for all cylinder sets, it means that every event in $\mathfrak{A}$ is contained in a set of measure zero.

It is the purpose of this work to lay some of the groundwork for future investigations into physically realistic examples for which the histories Hilbert space is infinite dimensional. These include the Schrodinger particle and the quantum random walk [4, 9. The existence of an extension of the quantum vector pre-measure in this infinite dimensional case requires a weaker condition than bounded variation, but there are indications that even this is not satisfied for the Schrodinger particle 20. This may suggest that time of passage questions, notoriously difficult to pose in the standard approach to quantum theory, are not observables even within the quantum measure approach.

Acknowledgements: We thank Rafael Sorkin and Bob Geroch for useful discussions. This work was supported in part by the Royal Society International Joint Project 2006-R2. 


\section{References}

[1] R. D. Sorkin, Mod. Phys. Lett. A 9, 3119 (1994) arXiv:gr-qc/9401003.

[2] R. D. Sorkin, "Quantum measure theory and its interpretation", 4TH Drexel Symposium on Quantum Nonintegrability, 8-11 Sep 1994, Philadelphia, PA, arXiv:gr-qc/9507057.

[3] R. B. Salgado, Mod. Phys. Lett. A 17, 711 (2002) arXiv:gr-qc/9903015.

[4] X. Martin, D. O'Connor and R. D. Sorkin, Phys. Rev. D 71, 024029 (2005), arXiv:gr-qc/0403085.

[5] S. Surya and P. Wallden, Found. Phys. 40: 585-606 (2010) arXiv:0809.1951.

[6] S. Gudder, J. Math. Phys. 50, 123509 (2009) arXiv:0909.2203.

[7] Mark Kac, "Probability and related topics in physical sciences", Lectures in applied mathematics, AMS Bookstore, 1957.

[8] Paul R. Halmos, "Measure Theory (Graduate Texts in Mathematics) (v. 18)", Springer, 1978.

[9] H. F. Dowker, S. Johnston and R. Sorkin, J. Phys. A 43, 275302 (2010), arXiv:1002.0589.

[10] J. Diestel and J. J. Uhl, "Vector Measures", Mathematical Surveys, Number 15, American Mathematical Society, 1977.

[11] I. Kluvnek, Proc. Am. Math. Soc. 81, No. 2, 233-239 (1981), Y. Klinen, Studia Mathematica 104 (3) 269-278 (1993), I. Dobrakov. Czech. Math. J., 37 (112), 487506. (1987).

[12] W. Rudin, "Real and Complex Analysis", McGraw-Hill International Editions, 1987.

[13] L. Bombelli, J. H. Lee, D. Meyer and R. Sorkin, Phys. Rev. Lett. 59, 521 (1987).

[14] D. P. Rideout and R. D. Sorkin, Phys. Rev. D 61, 024002 (2000) arXiv:gr-qc/9904062.

[15] D. P. Rideout,, "Dynamics of causal sets," PhD. Thesis May 2001, Syracuse University, arXiv:gr-qc/0212064.

[16] G. Brightwell, H. F. Dowker, R. S. Garcia, J. Henson and R. D. Sorkin, Phys. Rev. D 67, 084031 (2003) arXiv:gr-qc/0210061.

[17] R. Penrose, Techniques of Differential Topology in Relativity,(1972); S. Hawking \& G. Ellis, Large scale structure of spacetime. 
[18] R. D. Sorkin, J. Phys. Conf. Ser. 67, 012018 (2007) arXiv:quant-ph/0703276.

[19] F. Dowker and Y. Ghazi-Tabatabai, J. Phys. A 41, 105301 (2008) arXiv:0711.0894 [quant-ph]].

[20] R. Geroch, "Path Integrals", Unpublished notes available at http://physics.syr.edu/ sorkin/lecture.notes/ 